\title{
Characterization of Stripline Circuit by Planar Circuit/Equivalent Network and Demonstration of its Validity by 1D, 2D and 3D Analysis for Practical Structures \\ Jui-pang Hsu Takaharu Hiraoka and Takahiro Ogawa \\ Kanagawa University and MEL Inc.
}

\begin{abstract}
Stripline circuit can be modelled to planar circuit, whose equivalent network are rigorously given by modal analysis. Thus derived equivalent network can be analyzed by conventional circuit theory. Validity of "planar circuit/equivalent network model" is demonstrated by 1D, 2D and 3D analysis for practical structures.
\end{abstract}

\section{Introduction}

Stripline circuit is an important circuit structure for integration of microwave circuit. Hence, efficient calculation method is strongly needed for circuit analysis and design . These works have so far been done by EM simulator or Circuit simulator. EM simulator is constructed by FEM, MOM or FDTD, but these methods require so much cpu time and memory. While, circuit simulator is an efficient tool for calculation of circuit response, but its range of use is restricted because of the limitation on equivalent network used. In order to improve these situation, we propose " planar circuit/equivalent network model". Stripline circuit can be transformed to planar circuit by approximating stripline by planar waveguide. Planar circuit can be divided into three key elements, whose equivalent networks are rigorously derived by modal analysis. Hence, whole equivalent network of any stripline circuit can be given by combination of these three key elements, whose characteristics can be calculated by conventional circuit theory. Stripline circuit has three dimensional field, which means that 2D model of planar circuit/equivalent network has always some discrepancy. This discrepancy is estimated by 3D MOM for practical stripline circuit shown in Fig.1. It is demonstrated that the discrepancy between $2 \mathrm{D}$ and $3 \mathrm{D}$ results is relatively small, which means that present method of planar waveguide/ equivalent network model is valid and useful.

\section{Modelling of stripline circuit to planar circuit}

Stripline circuits such as shown in Fig. 1 are originally designed by $1 \mathrm{D}$ transmission line theory, where circuit parameters are specified by characteristic impedance and electrical length of constituting transmission line . Actual stripline dimension is given by translating these circuit parameters to practical dimension. Thus derived stripline circuit can be analyzed three-dimensionally by FEM, MOM or FDTD, but these methods consume so much cpu time and memory for good results. Instead we propose planar circuit/equivalent network model. Stripline can be modelled by planar waveguide with magnetic side wall as shown in Fig. $1^{[1]}$, where effective width is given by eq(1).

$$
\Delta=\frac{2}{\pi} \ln 2 \cdot d
$$

Then, the stripline circuit becomes planar waveguide circuit or planar circuit. Thus derived planar circuit can be analyzed again by 2D-FEM, MOM or FDTD, but we propose a rigorous equivalent network based on modal analysis.

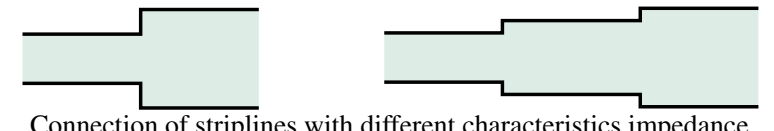

ines with different characteristic (a) Without impedance transformer (b) With impedance transformer
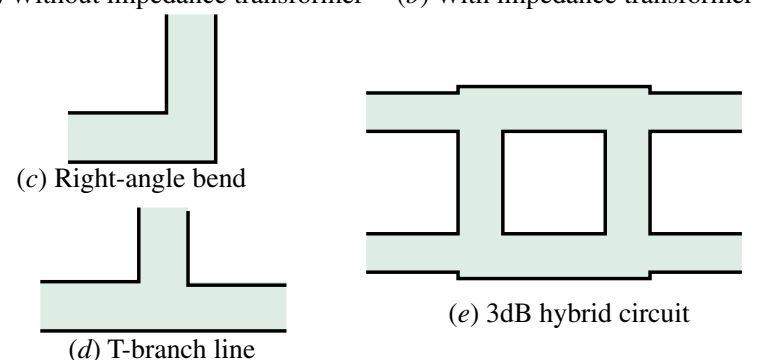

(e) $3 \mathrm{~dB}$ hybrid circuit

Fig.1 Various stripline circuits treated in this paper

3. Equivalent network for key elements/whole circuit Each part of thus derived planar circuit can be classified into three key elements, i.e., waveguide, step junction and planar junction. Equivalent network for these three key elements are given by multi-transmission line, multi-port ideal transformer and Foster-type equivalent network ${ }^{[2][3]}$, respectively, as shown in Fig.3 where simple vector representation is introduced in bold line. Circuit constant for each key element is given in the quoted references ${ }^{[2][3]}$.

Whole equivalent network for any stripline circuit is given by connecting equivalent network of key elements according to the topology of the stripline structure. Circuit response can be calculated by applying conventional circuit theory to thus derived equivalent network. This method is similar to circuit simulator, but more general and powerful because higher mode propagation is allowed and discontinuity problem is automatically taken into consideration by solving the corresponding eigenmode problem. In addition, field distribution at any instant can be calculated by excited mode amplitudes and eigenmodes.

\section{Practical 1D, 2D and 3D analysis for five structures}

In order to demonstrate the validity and usefulness of planar circuit/equivalent network model(2D), planar waveguide circuit is designed by using following characteristic impedance of planar waveguide.

$$
Z_{C}=\frac{1}{2} \sqrt{\frac{\mu_{0}}{\varepsilon}} \frac{d}{W}
$$

Frequency characteristics of stripline circuits shown in

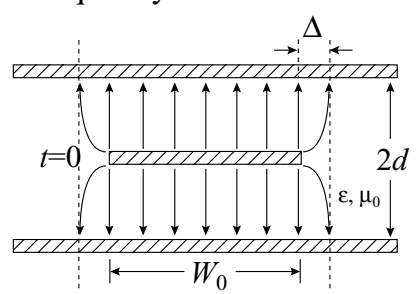

(a) Stripline

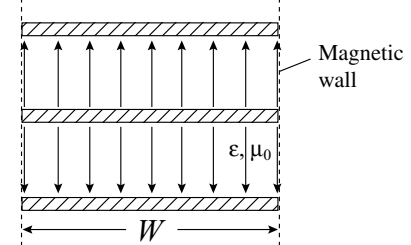

(b) Planar waveguide $\left(W=W_{0}+2 \Delta\right)$

Fig.2 Stripline and Planar waveguide model 
Fig. 1 are calculated by 1D transmission line theory(1Danalysis), 2D planar waveguide model/equivalent network(2D-analysis) and MOM analysis(3D-analysis). $2 \mathrm{D}$ results are compared with desired characteristics given by transmission line theory(1D) and with practically realized characteristics estimated by MOM analysis(3D). Former comparison shows how far the realized 2D results removes from the desired characteristics(1D) and latter comparison shows how exactly the calculated results by 2D approximate 3D results. The calculated results for five structures are explained in the following. (Relative dielectric constant $\varepsilon_{r}$ is assumed to be 2.62 through the analysis)

\section{1) Connection of $50[\Omega]$ and $30[\Omega]$ striplines}

Frequency characteristics for direct connection of two striplines with different characteristic impedance and that for reflectionless connection with impedance transformer are calculated by $1 \mathrm{D}, 2 \mathrm{D}$ and $3 \mathrm{D}$ analysis and shown in Fig.4. It is interesting that characteristics for direct connection is frequency independent for 1D analysis, but dependent for $2 \mathrm{D}$ and $3 \mathrm{D}$ analysis due to the frequency dependent field disturbance at discontinuity. 2D and 3D results for reflectionless case agree well, but different a little from $1 \mathrm{D}$ desired results. Through $2 \mathrm{D}$ analysis 30 waveguide modes are considered at widest planar waveguide.

\section{2) Stripline right-angle bend}

Frequency characteristics of stripline right-angle bend for three different characteristic impedance are calculated by $2 \mathrm{D}$ and $3 \mathrm{D}$ analysis and shown in Fig.5. Comparison between $2 \mathrm{D}$ and $3 \mathrm{D}$ results shows that the lower the characteristic impedance, the better the agreement. Because lower characteristic impedance realizes better planar circuit. Foster-type equivalent network parameters are calculated by analytically available eigenmodes for squareshaped planar junction. 100 planar-modes are considered.

\section{3) Stripline T-branch line $(25[\Omega]$ to $50[\Omega]$ )}

Frequency characteristics for stripline T-branch line shown in Fig. 6 are calculated by 1D, 2D and 3D analysis and shown in the same figure. The characteristics by 1D analysis are frequency-independent, but those by $2 \mathrm{D}$ and 3D analysis dependent. 2D and 3D results agree well at lower frequency, but not at higher frequency because of extra coupling neglected in 2D analysis. As for 2D analysis, 200 planar-modes are considered in rectangular junction.

\section{4) Branch-line 3dB hybrid circuit}

Multimode equivalent network for branch-line 3dB hybrid circuit is shown in Fig.7(c), where \#5 and \#8 are treated as wide but short waveguides. 50 waveguide modes are considered at waveguide \#5 and \#8. Dimension and frequency characteristics by $2 \mathrm{D}$ and $3 \mathrm{D}$ analysis for three different center frequency cases are shown in Fig.7. Ideal characteristics are realized by circuit of lower center frequency, but not by that of higher center frequency. It may be due to the relatively larger area of planar junction compared with dimension of waveguide for higher center frequency. However, agreement between $2 \mathrm{D}$ and $3 \mathrm{D}$ results for three cases are relatively good as seen from Fig.7.

\section{Conclusion}

Planar circuit/equivalent network model is proposed for stripline circuit. Wide-band frequency characteristics and field distribution at operation can be calculated by applying conventional circuit theory to the equivalent network. Thus calculated wide band frequency characteristics (2Danalysis) are compared with that based on transmission line theory(1D-analysis) and MoM.(3D-analysis). Relatively good agreement between $2 \mathrm{D}$ and 3D calculated results means that "planar circuit/equivalent network model" is working well, and so valid and useful.

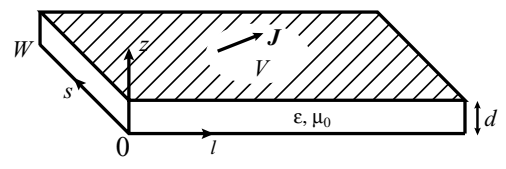

(a) Planar waveguide

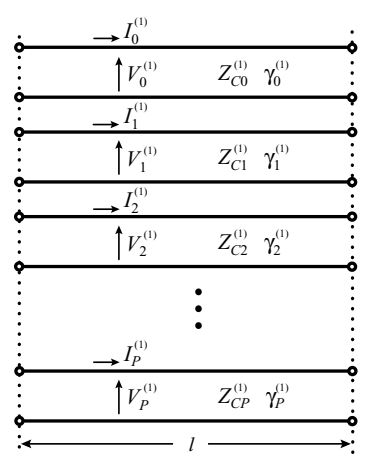

(b) Multi-transmission line

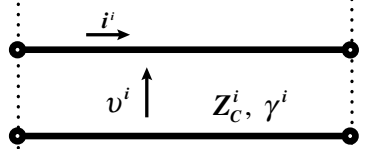

(c) Vector representation

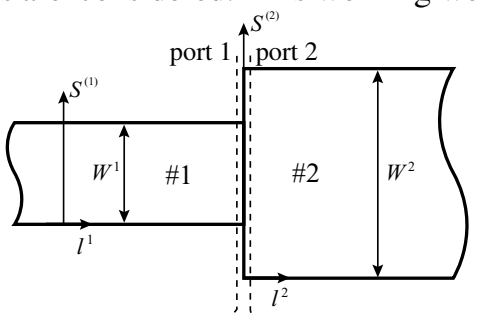

(a) Step junction

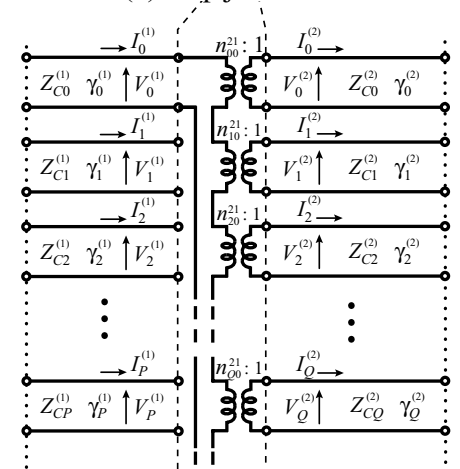

(b) Multi-port ideal transformer

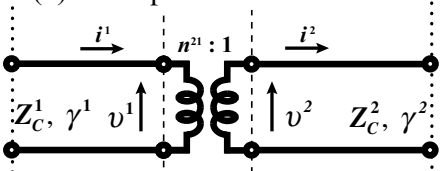

(c) Vector representation
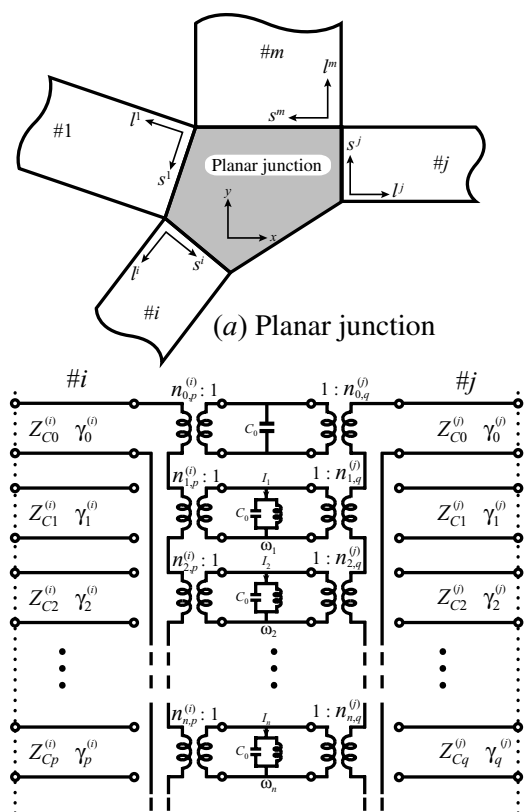

(b) Foster-type equivalent network

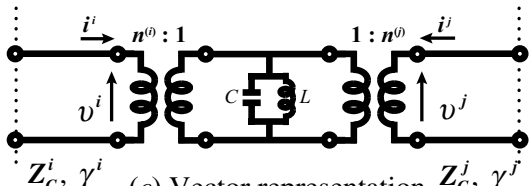

(c) Vector representation $Z_{C}^{j}, \gamma^{j}$

Fig.3 Three types of key element, their equivalent networks and vector representation in bold line 


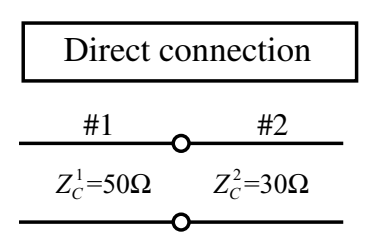

(a) Transmission line circuit

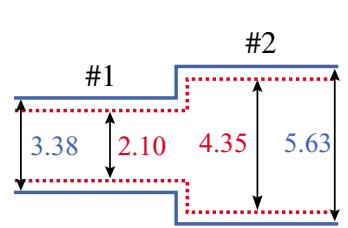

(b) 2D shape $[\mathrm{mm}]$

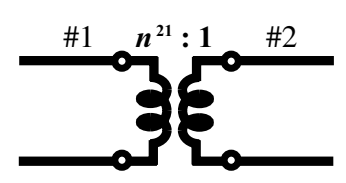

(c) Equivalent network
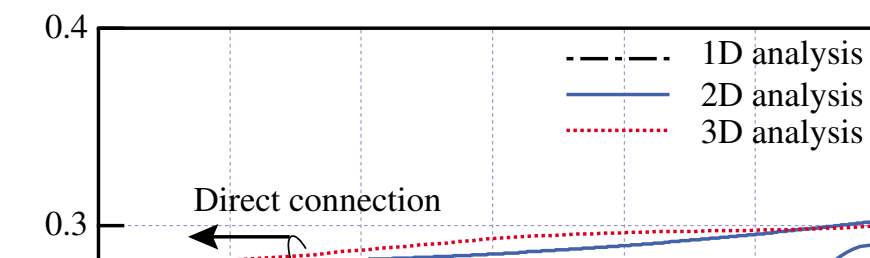

Direct connection

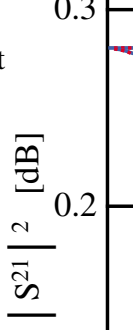

0.1

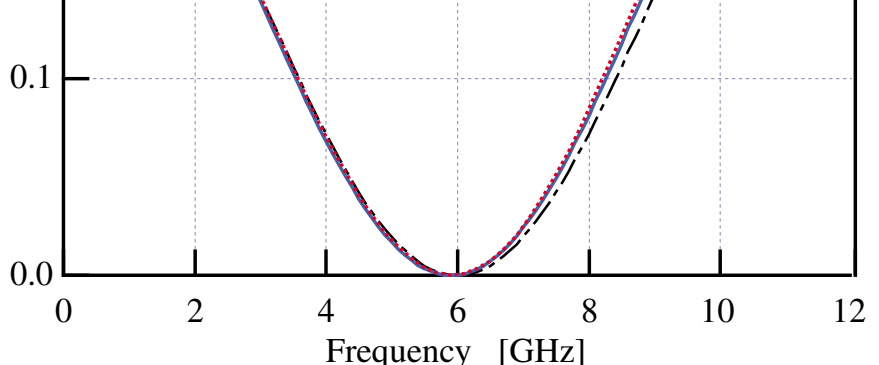

No reflection connection

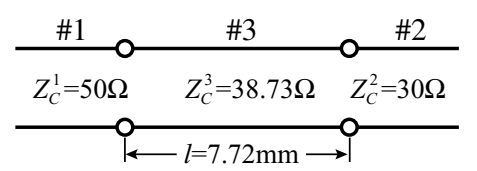

(a)' Transmission line circuit

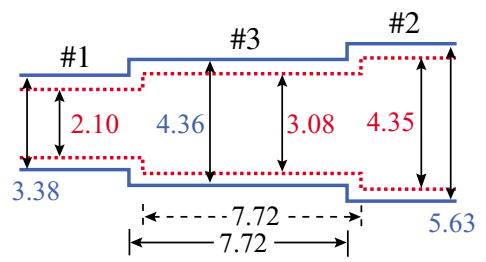

(b)' 2D shape [mm]

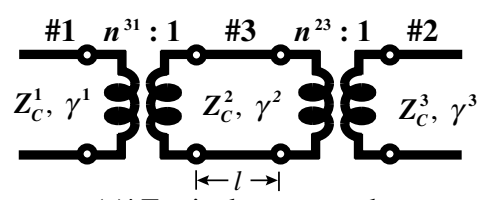

(c)' Equivalent network

$(a) /(a)^{\prime} 1 \mathrm{D}$ transmission line circuit to be realized, $\left.(b) /(b)\right)^{\prime} 2 \mathrm{D}$ shape of the corresponding stripline circuit (dotted line) and planar waveguide circuit(solid line) with dimension, $(c) /(c)^{\prime}$ Multi-mode equivalent network in vector representation

Fig.4 Direct and reflectionless connection of two striplines with different characteristic impedance — structures, equivalent network for 2D model and frequency characteristics by 1D, 2D and 3D analysis -

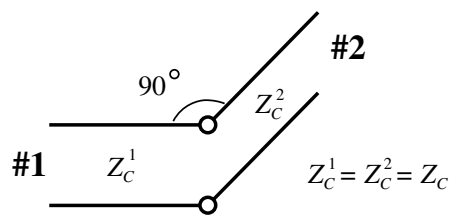

(a) Right-angle bend

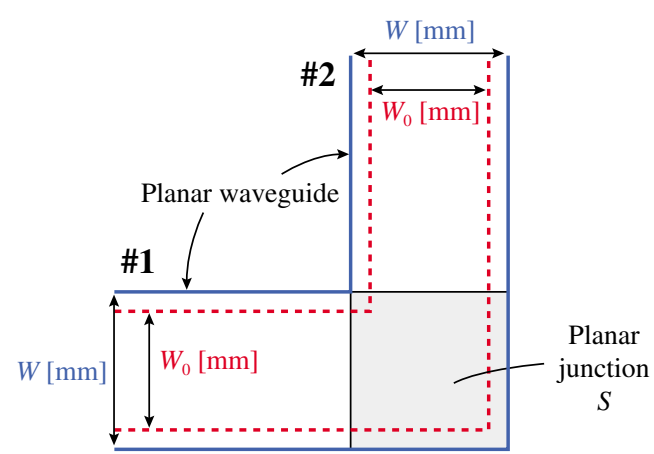

(b) 2D shape of planar waveguide and stripline

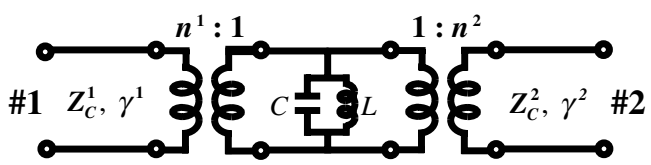

(c) Equivalent network in vector representation

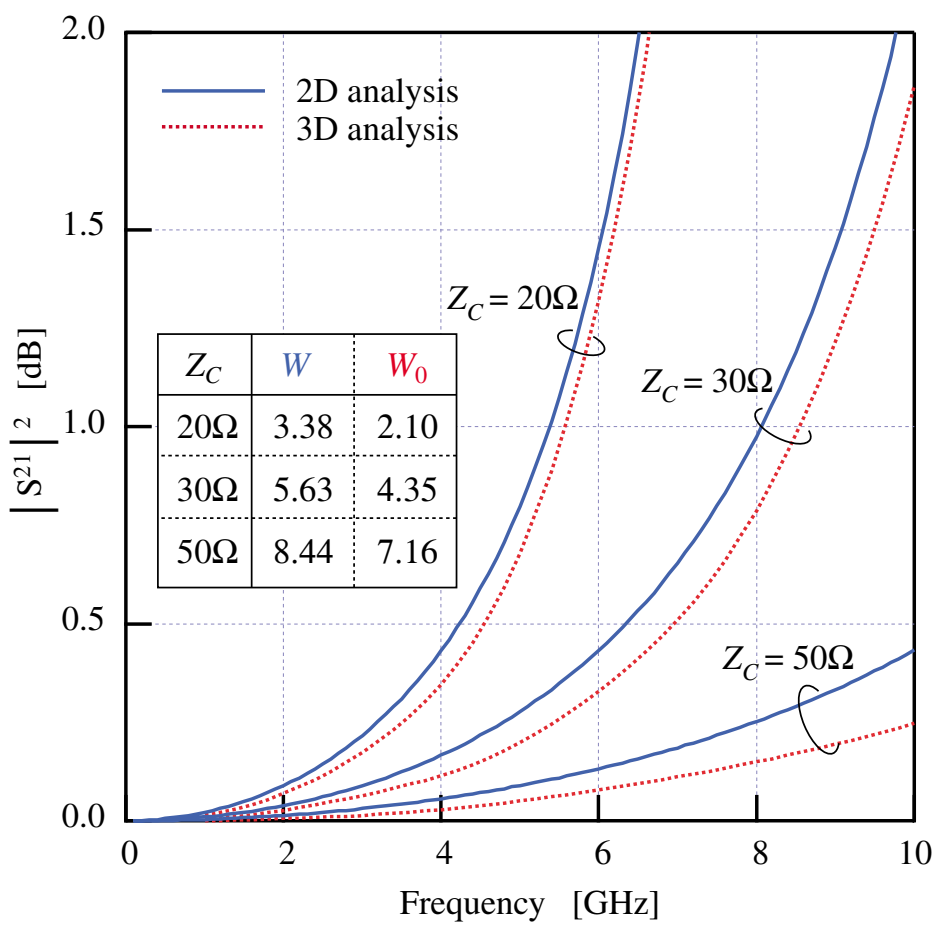

(a) 1D transmission line circuit to be realized, (b) 2D shape of the corresponding stripline circuit (dotted line) and planar waveguide circuit(solid line) with dimension, (c) Multi-mode equivalent network in vector representation

Fig.5 Right-angle bend with different characteristic impedance of $20[\Omega], 30[\Omega]$ and $50[\Omega]$ — structures, equivalent network for 2D model and frequency characteristics by 2D and 3D analysis -

Reference [1] Oliner, A.A, "Equivalent circuits for discontinuities in balanced strip transmission line", IRE, MTT.,vol.3, No.2, pp134-143, March 1955 [ 2] Hsu, Jui-Pang "Analysis of planar circuit by eigenfunction expansion", Analysis method for electomagnetic ploblems edited by E. Yamashita, Vol.2, Sec.6.1, pp226-251 Artech House, 1995 [3] Hsu,J.P, Hiraoka,H, Tabei,Y,"Analysis of stripline right angle bend with slant-wise corner cut based on eigenmode expansion method and Foster-type equivalent network", 1995 IEEE MTT-S IMS Digest pp1315-1318, June 1999 


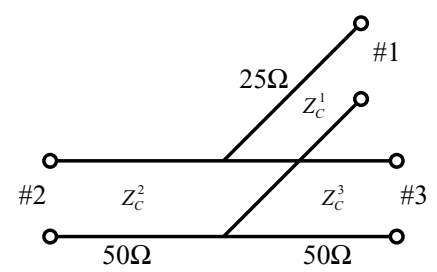

(a) $1 \mathrm{D}$ transmission line

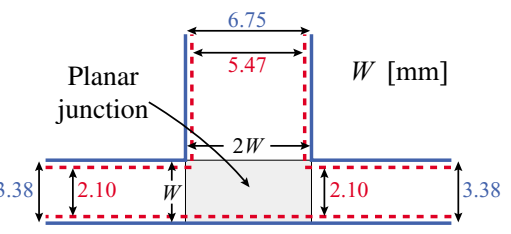

(b) 2D shape of stripline and planar waveguide

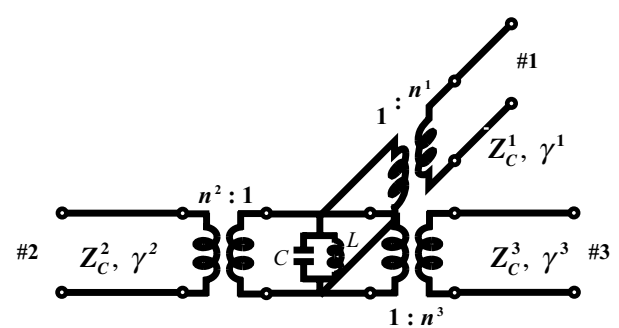

(c) Equivalent network in vector representation

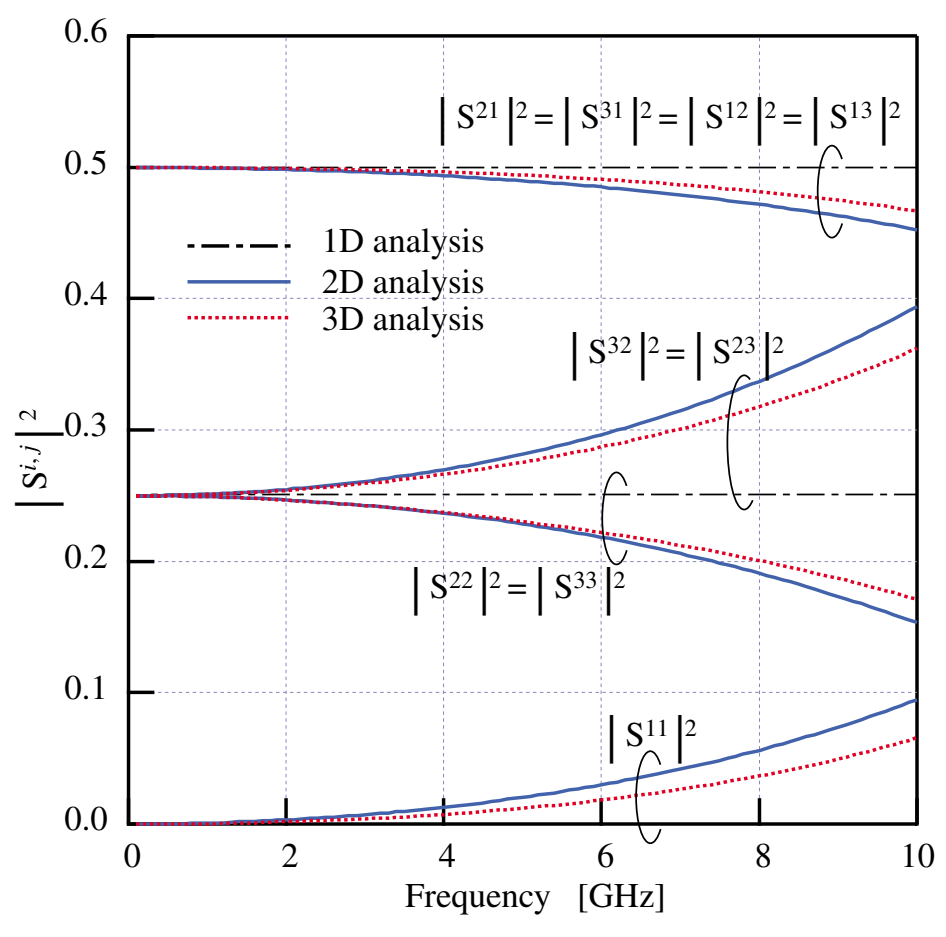

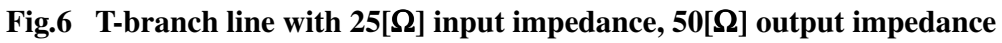
- structures, equivalent network for 2D model and frequency characteristics by 1D, 2D and 3D analysis -

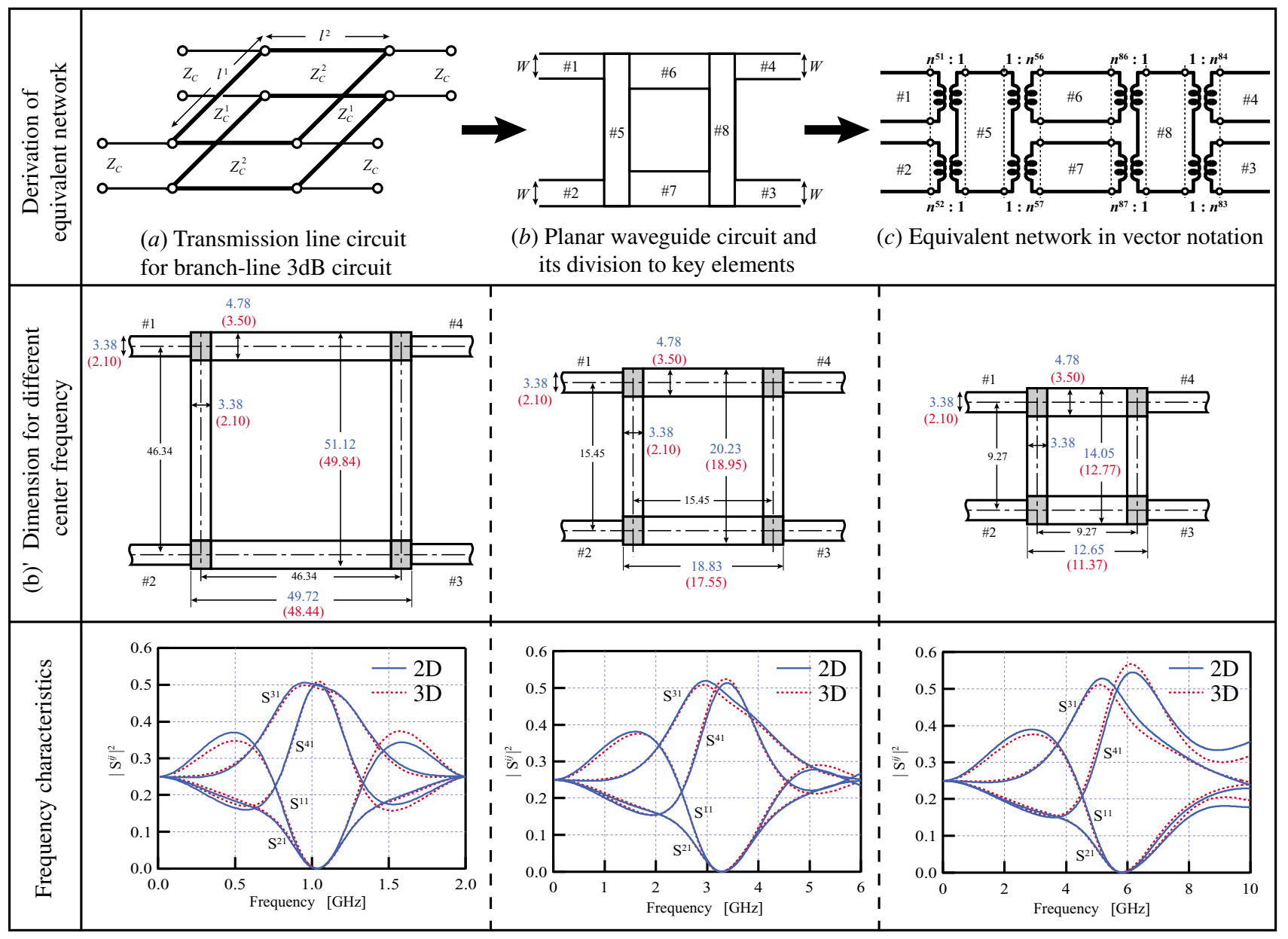

(a) 1D transmission line circuit to be ralized, $(b) /(b)$ ' 2D shape of stripline circuit and planar waveguide circuit with dimension where ( ) is dimension for stripline circuit, $(c)$ Multimode equivalent network in vector representaion

Fig.7 Branch-line 3dB hybrid circuit for $1 \mathrm{GHz}, 3 \mathrm{GHz}$ and $5 \mathrm{GHz}$ center frequency

— structures, equivalent network for 2D model and frequency characteristics by 2D (solid line) and 3D (dotted line)analysis - 